\title{
NONLINEAR ATTITUDE AND GYROSCOPE'S BIAS ESTIMATION FOR A VTOL UAV
}

\author{
Jean Michel Pflimlin ${ }^{*, 1}$ Tarek Hamel** \\ Philippe Souères* Najib Metni ${ }^{* * *}$ \\ * LAAS - CNRS, 7, Av. du colonel Roche, 31077 Toulouse \\ ** I3S - CNRS, Les Algorithmes, Bât. Euclide, 2000, Rte \\ des Lucioles B.P.121, 06903 Sophia Antipolis \\ *** LCPC, 58, Bvd. Lefebvre, 75732 Paris Cedex 15
}

\begin{abstract}
This paper addresses the problem of attitude and heading restitution for a VTOL UAV. We describe an observation strategy to restitute the complete attitude matrix of the vehicle starting from Inertial Measurement Unit (IMU) and magnetometers. This study is a part of the development of the ducted fan UAV designed by Bertin Technologies. First, a measured orientation matrix is calculated from both inertial vectors which are the gravity and the earth magnetic field. Then, an estimated orientation is built by integrating gyroscopic readings, and corrected by the measured one. Nonlinear observation techniques are used to design a nonlinear estimator of the orientation matrix and an adaptive filter of the gyroscope's bias which ensure the convergence of the observer. Such an observer is as efficient as classical Extended Kalman Filtering based observers, and easier to implement in real time. Simulations are proposed to illustrate the concept. Copyright $^{\circledR}$ 2005 IFAC
\end{abstract}

Keywords: VTOL UAV, Attitude and Heading Restitution Sensor, SO(3) group

\section{INTRODUCTION}

The design of autonomous navigation strategy for Micro Air Vehicles (MAV) has now become a very challenging research area. Those small and discreet flying vehicles, able to perform vertical takeoff and landing (VTOL), are of great interest for civil and military operations in urban environment. The design of autonomous VTOL vehicle capable of stationary flight has recently motivated many research projects (Pflimlin et al., 2004). Significant interest has been directed towards the development of autonomous scale model helicopter due to their high payload to power ratio. Helicopters, however, are extremely dangerous in practice due to the exposed rotor blades. Very

1 Partially supported by Bertin Technologies, and the French ministry of Armament little has been done to the development of secure platforms (Hamel et al., 2002). Such platforms have a considerable potential for surveillance and inspection roles in dangerous or awkward environments. One of the projects of the French National Direction of Armament, DGA, is to supply the infantry with such micro-drones by 2007. As a part of this project, the group Bertin Technology has in charge the development of a ducted fan UAV which is represented in figure 1. The work presented in this paper has been developed in the framework of a collaboration between, Bertin Technologies, the LAAS-CNRS and I3S laboratories, both devoted to modelling and elaboration of autonomous control strategies. In a first step, the automatic control design must allow the vehicle to be easily operated by an inexperienced user, despite wind perturbations. Designing such a remote 


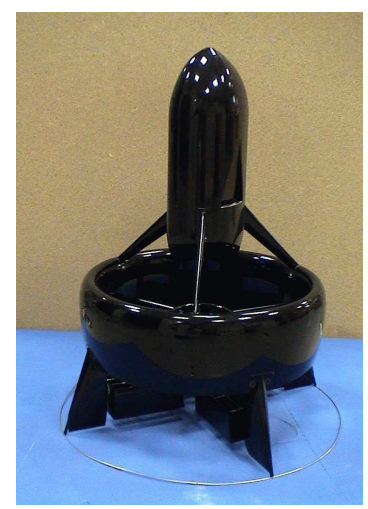

Fig. 1. A view of Bertin VTOL UAV.

control requires an efficient attitude control, able to deal with internal coupling of the system and to maintain the vehicle in a secure flight envelope. This is strongly dependent on a reliable attitude estimation. Much has been done in attitude and heading restitution using Extended Kalman filters and a representation of the orientation based on quaternions (Marins et al., 2001). However, implementing a Kalman filter pose serious problem due in particular to the high sensitivity of the covariance matrix to numerical errors. Engineering techniques such as $[U, D]$-decomposition are indispensable in practice to increase robustness of the filter (Grewal et al., 2001). Using rotation matrices to describe the orientation and designing a nonlinear observer directly in the rotation set $S O(3)$ allows to overcome the linearization step and to provide a more physical view of what the observer is doing.

In this paper we describe an observation strategy to restitute the complete attitude matrix of the micro-drone starting from Inertial Measurement Unit (IMU) and magnetometers. The proposed observer is as efficient as an Extended Kalman filter and much easier to implement. The paper is organized as follows: section 2 provides a description of the geometric and kinematic model of the attitude and a model of the sensors used to provide the attitude measurements. The observer design is given in section 3. Simulation results are then proposed to illustrate the method.

\section{SYSTEM MODELLING}

\subsection{Attitude representation and kinematics}

Two reference frames are considered to model the system (Boiffier, 1998):

- $\mathcal{I}$ is an inertial frame attached to the earth. It is assumed to be Galilean. It is associated to the vector base $\left\{x_{0}, y_{0}, z_{0}\right\}$, where $x_{0}$ points to the magnetic North, $z_{0}$ points to the center of earth, and $y_{0}$ points to the East to complete the base.

- $\mathcal{A}$ is body-fixed frame attached to the vehicle. It is associated to the vector base $\left\{x_{b}, y_{b}, z_{b}\right\}$. The conventional roll, pitch and yaw axis used in mechanics of flight are considered in this paper: $x_{b}$ points forward, $z_{b}$ downward, and to complete the base, $y_{b}$ points to the right.

The transformation matrix between the inertial frame and the body fixed frame is called attitude matrix $R$. By definition, each column of $R$ is made of the components of the corresponding body fixed base vector expressed in the inertial frame ${ }^{2}$ :

$$
R=\left[x_{b}, y_{b}, z_{b}\right]_{\mathcal{I}}
$$

$R$ being the transformation matrix between two orthogonal frames, it is an element of $S O(3)$. With the usual matrix product, $S O(3)$ is a group whose elements satisfy (Murray et al., 1994):

$$
\forall P \in S O(3), P^{-1}=P^{T} \text { et } \operatorname{det}(P)=1
$$

Introducing the canonical base $\left\{e_{1}, e_{2}, e_{3}\right\}$ of $\mathbb{R}^{3}$ : $e_{1}=[1,0,0]^{T}, e_{2}=[0,1,0]^{T}, e_{3}=[0,0,1]^{T}$, the components of the inertial base vectors with respect to the body fixed frame are given by:

$$
\left[x_{0}\right]_{\mathcal{A}}=R^{T} e_{1} \quad\left[y_{0}\right]_{\mathcal{A}}=R^{T} e_{2} \quad\left[z_{0}\right]_{\mathcal{A}}=R^{T} e_{3}
$$

Denoting by $\Omega=[p, q, r]^{T}$ the angular velocity vector of the body fixed frame relative to the inertial frame, expressed in body frame, the kinematic equation of attitude that expresses the evolution of $R$ through time is given by (Murray et al., 1994):

$$
\dot{R}=R \operatorname{sk}(\Omega)
$$

Where $\operatorname{sk}(\Omega)$ denotes the skew-symmetric matrix $^{3}$ associated to $\Omega$.

\subsection{IMU and magnetometers modelling}

The VTOL UAV is equipped with a low cost strap down IMU, that provides the angular velocity $\Omega$ components as well as the linear accelerations, and a 3-axis magnetometer. Angular rates allow to predict the evolution of the attitude matrix, while the measure provided by accelerometers and magnetometers is used to correct this prediction. When designing an observer, one has to make a trade-off between the drift resulting from integration of the gyroscopic measurements and the reliability of noisy readings provided by accelerometers and magnetometers.

Gyroscope modelling The 3-axis gyroscope provides the angular velocities of the body frame relative to the inertial frame, expressed in the body fixed frame. If the measured angular rates were perfect, it would be sufficient to integrate these measurements using equation (1) to provide the orientation matrix $R$. However, due to openloop integrations, any bias on gyroscopic measurements lead to an error on the attitude prediction

\footnotetext{
2 To describe the attitude of a vehicle, many authors use the Direct Cosine Matrix DCM. By definition one gets $D C M=R^{T}$

${ }^{3} \forall u \in \Re^{3}, \operatorname{sk}(u)$ is the skew matrix associated to $u$ : $\forall v \in \Re^{3}, u \times v=\operatorname{sk}(u) v$
} 
which grows to infinity. This phenomena is known as gyroscopic drift. To take into account on-line estimation of gyroscope's bias, we consider the model of gyroscopic measurements given by:

$$
\Omega_{m}=\Omega+b
$$

where $b$ depends on the temperature. Many low cost IMU are now internally compensated in temperature, and as a result, $b$ oscillates slowly around a constant average value. In the observer design, $b$ will be supposed constant. We will propose an adaptive filter to estimate $b$ on-line. In practice, the estimator will succeed in estimating gyroscope's bias even though they vary slowly.

Accelerometers modelling The 3-axis accelerometer provides the non gravitational linear accelerations applied to the vehicle relative to inertial frame (Radix, 1980). Accelerometers readings gather all forces applied to the vehicle, except gravity. In case of "strap-down" IMU, readings are expressed in body fixed frame. When the absolute acceleration of the vehicle in the inertial frame is weak $\left(\left\|\gamma_{a b s}\right\| \ll g\right)$, accelerometers' readings $A_{m}$ provide the components of gravity vector in $\mathcal{A}$ :

$$
\overrightarrow{A_{m}}=\underbrace{\gamma_{a b s}}_{\left\|\boldsymbol{\gamma}_{a b s}\right\| \ll g}-g z_{0} \Longrightarrow A_{m} \approx-g R^{T} e_{3}
$$

If measurements were absolutely reliable, pitch and roll of the vehicle could be directly deduced from the accelerometers, working as inclinometers in that case. However, accelerometers' readings are very noisy and very sensitive to the vibratory environment surrounding the IMU. Furthermore, if for any reason (wind gust, or transition from hovering to forward flight), the vehicle acquires a non negligible absolute acceleration in the inertial frame, the attitude provided by accelerometers will no more be reliable. Therefore, it is important in practice to correlate the attitude measured by the accelerometers and the attitude predicted by integrating the gyroscopic measurements to provide a correct attitude estimation (Suh, 2003).

Magnetometers modelling To achieve complete attitude estimation, we add to inertial measurements the information provided by a 3 -axis magnetometer. Such a device is able to give the yaw angle $\psi$ by measuring the earth magnetic field in the body fixed frame. Let $\boldsymbol{m}$ be the direct cosine vector of the earth magnetic field. Let $m$ and $h$ be the components of $\boldsymbol{m}$ respectively in the inertial frame $\mathcal{I}$ and in the body fixed frame $\mathcal{A}$. Then we have the following equation:

$$
h=R^{T} m
$$

By definition of the inertial frame, one gets $m \in$ $\operatorname{span}\left\{e_{1}, e_{3}\right\}$, that is to say:

$$
m=\left(e_{1}^{T} m\right) e_{1}+\left(e_{3}^{T} m\right) e_{3}
$$

\subsection{Measured orientation matrix construction}

Both inertial vectors measured by the IMU completed with magnetometers are represented in figure 2. Let $u$ be the normalized components of the

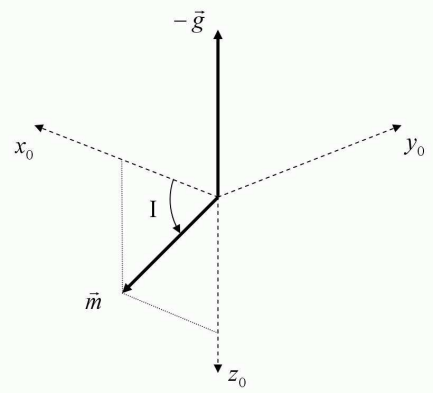

Fig. 2. Representation of the gravity and the earth magnetic field in the inertial frame.

accelerometers readings in body frame:

$$
u=\frac{A_{m}}{\left\|A_{m}\right\|} \approx-R^{T} e_{3}
$$

We are going to present a method to build the complete attitude matrix $R$ by using both inertial vectors $\boldsymbol{m}$ and $\boldsymbol{g}$ expressed in body frame. The main idea is to use Gram-Schmidt's orthonormalization method to construct $R^{T} e_{1}$ and $R^{T} e_{3}$ starting from $h$ and $u$ and to complete the base by the cross product of both vectors.

Proposition 1. Consider the following matrix $\mathcal{R}$ built from $h$ and $u$ :

$$
\mathcal{R}=\left[\begin{array}{ll}
\frac{h-\left(u^{T} h\right) u}{\left\|h-\left(u^{T} h\right) u\right\|} & \frac{-u \times h}{\left\|h-\left(u^{T} h\right) u\right\|}-u
\end{array}\right]^{T}
$$

Then we have $\mathcal{R}=R$.

Proof. First we have:

$$
\begin{aligned}
h-\left(u^{T} h\right) u & =R^{T} m-\left(-\left(R^{T} e_{3}\right)^{T} R^{T} m\right)\left(-R^{T} e_{3}\right) \\
& =R^{T}\left(m-\left(e_{3}^{T} m\right) e_{3}\right) \\
& =\left(e_{1}^{T} m\right) R^{T} e_{1}
\end{aligned}
$$

By convention $e_{1}$ points to the North, so $e_{1}^{T} m>0$. It yields:

$$
e_{1}^{T} m=\left\|h-\left(u^{T} h\right) u\right\|
$$

So the first column of $\mathcal{R}^{T}$ expresses as $R^{T} e_{1}$. The second column of $\mathcal{R}^{T}$ can be written:

$$
\frac{-u \times h}{\left\|h-\left(u^{T} h\right) u\right\|}=(-u) \times \frac{h-\left(u^{T} h\right) u}{\left\|h-\left(u^{T} h\right) u\right\|}=\left(R^{T} e_{3}\right) \times\left(R^{T} e_{1}\right)
$$

As $\left(R^{T} e_{3}\right) \times\left(R^{T} e_{1}\right)=R^{T}\left(e_{3} \times e_{1}\right)$, the second column of $\mathcal{R}^{T}$ equals $R^{T} e_{2}$. Finally, we get:

$$
\mathcal{R}^{T}=R^{T}\left[e_{1}, e_{2}, e_{3}\right]=R^{T}
$$

\section{OBSERVER DESIGN}

In this section, a nonlinear estimator of the orientation matrix and an adaptive filter of the gyroscope's bias are designed. Convergence of the 
resulting estimator is achieved by means of nonlinear observation techniques (Krstic et al., 1995). Let us denote by $\hat{R} \in S O(3)$ and $\hat{b}$ the estimations of $R$ and $b$ respectively. Estimations errors $\tilde{R}$ and $\tilde{b}$ are defined by:

$$
\tilde{R}=\hat{R}^{T} R \text { and } \tilde{b}=b-\hat{b}
$$

The convergence of the observer is ensured if and only if the following two conditions are verified:

$$
\tilde{R} \longrightarrow I_{3 \times 3} \text { and } \tilde{b} \longrightarrow 0
$$

The observer design consists in building filters on $\dot{\hat{R}}$ and $\dot{\hat{b}}$. To keep $\hat{R}$ within $S O(3)$, the following structure on the estimator is imposed:

$$
\dot{\hat{R}}=\hat{R} \operatorname{sk}(\hat{\Omega}) \text { and } \dot{\hat{b}}=\Gamma \tau
$$

Where $\Gamma$ is a positive gain which allows to tune up the adaption of the gyroscope's bias.

Let us introduce the following direct cosine vectors of $\mathbb{R}^{3}$, which represent the components of the inertial base vectors in the body frame and in the estimated body frame respectively:

$$
\begin{aligned}
& x=R^{T} e_{1} ; y=R^{T} e_{2} ; z=R^{T} e_{3} \\
& \hat{x}=\hat{R}^{T} e_{1} ; \hat{y}=\hat{R}^{T} e_{2} ; \hat{z}=\hat{R}^{T} e_{3}
\end{aligned}
$$

Let $X, Y, Z$ denote the cross product between the estimated direct cosine vectors and the real ones: $X=\hat{x} \times x, Y=\hat{y} \times y, Z=\hat{z} \times z$.

Theorem 1. With the above notations, the estimation law on attitude $\hat{\Omega}$ given by:

with $\varpi$, defined as:

$$
\hat{\Omega}=\tilde{R}\left(\Omega_{m}-\hat{b}\right)+\varpi
$$

$$
\varpi=-k(X+Y+Z), \quad k>0
$$

and the adaptive filter $\tau$ defined by:

$$
\tau=\tilde{R}^{T}(X+Y+Z)
$$

ensure convergence of the estimator given in (5) for any initial rotation angle $\alpha_{0}$ between the estimated orientation matrix and the real one and any initial estimation error on gyroscope bias $\tilde{b}_{0}$ verifying:

$$
\begin{aligned}
& \left|\tilde{b}_{0}\right| \leq b_{\max } \text { with } b_{\max } \leq 2 \sqrt{2 \Gamma} \\
& \left|\alpha_{0}\right|<\alpha_{\max } \text { with } \alpha_{\max }=\arccos \left(1-\frac{1}{2}\left(4-\frac{b_{\max }^{2}}{2 \Gamma}\right)\right)
\end{aligned}
$$

Proof. Let us define the Lyapunov function:

$$
V=\frac{1}{2}\left\|I_{3 \times 3}-\tilde{R}\right\|_{F r o b}^{2}+\frac{1}{2} \Gamma^{-1} \tilde{b}^{T} \tilde{b}
$$

In the expression of $V,\left\|I_{3 \times 3}-\tilde{R}\right\|_{F r o b}$ denotes the Frobenius norm ${ }^{4}$ of the matrix $I_{3 \times 3}-\tilde{R}$. Using the explicit form of this norm, it yields:

$$
V=\frac{1}{2} \operatorname{trace}\left(\left(I_{3 \times 3}-\tilde{R}\right)\left(I_{3 \times 3}-\tilde{R}\right)^{T}\right)+\frac{1}{2} \Gamma^{-1} \tilde{b}^{T} \tilde{b}
$$

4 Recall that the Frobenius norm of a square matrix $M$ is given by:

$$
\|M\|_{F r o b}=\sqrt{\operatorname{trace}\left(M M^{T}\right)}
$$

Developing the term $\left(I_{3 \times 3}-\tilde{R}\right)\left(I_{3 \times 3}-\tilde{R}\right)^{T}$, one gets

$$
\left(I_{3 \times 3}-\tilde{R}\right)\left(I_{3 \times 3}-\tilde{R}\right)^{T}=I_{3 \times 3}-\tilde{R}-\tilde{R}^{T}+\tilde{R} \tilde{R}^{T}
$$

As a product of two matrices in $S O(3), \tilde{R}$ is naturally in $S O(3)$. This means that $\tilde{R} \tilde{R}^{T}=I_{3 \times 3}$. So, as $\operatorname{trace}(\tilde{R})=\operatorname{trace}\left(\tilde{R}^{T}\right)$, the final expression of $V$ is given by:

$$
V=3-\operatorname{trace}(\tilde{R})+\frac{1}{2} \Gamma^{-1} \tilde{b}^{T} \tilde{b}
$$

Now, let us calculate the time derivative of $\tilde{R}$. Using (5), one gets:

$$
\begin{aligned}
\dot{\tilde{R}} & =\dot{\hat{R}}^{T} R+\hat{R}^{T} \dot{R}=-\operatorname{sk}(\hat{\Omega}) \hat{R}^{T} R+\hat{R}^{T} R \operatorname{sk}(\Omega) \\
& =-\operatorname{sk}(\hat{\Omega}) \tilde{R}+\tilde{R} \operatorname{sk}(\Omega)
\end{aligned}
$$

There, let us recall one of the main properties of skew-symmetric matrix (Murray et al., 1994):

$$
\forall R \in S O(3), \forall \omega \in \mathbb{R}^{3}, \quad R \operatorname{sk}(\omega) R^{T}=\operatorname{sk}(R \omega)
$$

So $\dot{\tilde{R}}$ is given by:

$$
\dot{\tilde{R}}=-\operatorname{sk}(\hat{\Omega}) \tilde{R}+\tilde{R} \operatorname{sk}(\Omega) \underbrace{I_{3 \times 3}}_{=\tilde{R}^{T} \tilde{R}}=-\operatorname{sk}(\hat{\Omega}-\tilde{R} \Omega) \tilde{R}
$$

Now, using (2), equation (6) can be written:

$$
\hat{\Omega}=\tilde{R}(\underbrace{\Omega_{m}}_{\Omega+b}-\hat{b})+\varpi=\tilde{R}(\Omega+\tilde{b})+\varpi
$$

It yields the final expression of $\dot{\tilde{R}}$ :

$$
\dot{\tilde{R}}=-\operatorname{sk}(\tilde{R} \tilde{b}+\varpi) \tilde{R}
$$

Now, let us go back to the calculation of $\dot{V}$. Using (10), one gets:

$$
\dot{V}=-\operatorname{trace}(\dot{\tilde{R}})+\Gamma^{-1} \tilde{b}^{T} \dot{\tilde{b}}
$$

As stated before, the gyroscope's bias is supposed constant. Therefore, $\dot{\tilde{b}}$ is simply $\dot{\tilde{b}}=-\dot{\hat{b}}$. Using the expression of $\dot{\tilde{R}}$ given by (11), $\dot{V}$ can be written:

$$
\dot{V}=\operatorname{trace}(\operatorname{sk}(\tilde{R} \tilde{b}+\varpi) \tilde{R})-\Gamma^{-1} \tilde{b}^{T} \dot{\hat{b}}
$$

At this stage, one can notice that $\tilde{R}$ can be expressed by:

$$
\begin{aligned}
\tilde{R} & =\hat{R}^{T} R=\hat{R}^{T}\left(e_{1} e_{1}^{T}+e_{2} e_{2}^{T}+e_{3} e_{3}^{T}\right) R \\
& =\left(\hat{R}^{T} e_{1}\right)\left(e_{1}^{T} R\right)+\left(\hat{R}^{T} e_{2}\right)\left(e_{2}^{T} R\right)+\left(\hat{R}^{T} e_{3}\right)\left(e_{3}^{T} R\right)
\end{aligned}
$$

That is to say:

$$
\tilde{R}=\hat{x} x^{T}+\hat{y} y^{T}+\hat{z} z^{T}
$$

So $\dot{V}$ is finally given by:

$$
\begin{aligned}
\dot{V}= & \operatorname{trace}\left(\operatorname{sk}(\tilde{R} \tilde{b}+\varpi) \hat{x} x^{T}\right)+\operatorname{trace}\left(\operatorname{sk}(\tilde{R} \tilde{b}+\varpi) \hat{y} y^{T}\right) \\
& +\operatorname{trace}\left(\operatorname{sk}(\tilde{R} \tilde{b}+\varpi) \hat{z} z^{T}\right)-\Gamma^{-1} \tilde{b}^{T} \hat{b}
\end{aligned}
$$

Let us recall the following property of the trace:

$$
\forall a, b \in \mathbb{R}^{3} \quad \operatorname{trace}\left(a b^{T}\right)=a^{T} b
$$

This property leads to the following expression:

$$
\begin{aligned}
\dot{V}= & {[\operatorname{sk}(\tilde{R} \tilde{b}+\varpi) \hat{x}]^{T} x+[\operatorname{sk}(\tilde{R} \tilde{b}+\varpi) \hat{y}]^{T} y } \\
& +[\operatorname{sk}(\tilde{R} \tilde{b}+\varpi) \hat{z}]^{T} z-\Gamma^{-1} \tilde{b}^{T} \hat{\hat{b}}
\end{aligned}
$$


Keeping in mind the basic properties ${ }^{5}$ of skewsymmetric matrices, the following expression of $\dot{V}$ is obtained:

$$
\begin{aligned}
\dot{V}= & (\tilde{R} \tilde{b})^{T}(\hat{x} \times x+\hat{y} \times y+\hat{z} \times z)-\Gamma^{-1} \tilde{b}^{T} \dot{\hat{b}} \\
& +\varpi^{T}(\hat{x} \times x+\hat{y} \times y+\hat{z} \times z)
\end{aligned}
$$

The adaptive filter given by (8) cancels the influence of $\tilde{b}$ in the expression of the Lyapunov function time derivative, which therefore can be written as follows:

$$
\dot{V}=\varpi^{T}(X+Y+Z)
$$

Finally, with the expression of $\varpi(7)$, we get:

$$
\dot{V}=-k|X+Y+Z|^{2}
$$

The non positivity of $\dot{V}$ is then ensured. Using the theorem of Lasalle, the observer state tends to the invariant set of $\dot{V}$ :

$$
\left\{(M, \hat{b}) \in S O(3) \times \mathbb{R}^{3} / X+Y+Z=0\right\}
$$

The resulting gap $\tilde{R}$ between the estimated orientation matrix and the real one defines a rotation of an angle $\alpha$ about a unit vector $\omega$ between $x, y, z$ and $\hat{x}, \hat{y}, \hat{z}$. In the base $\{x, y, z\}$, this rotation expresses as $\tilde{R}=\exp (\operatorname{sk}(\omega) \alpha) \triangleq\left[r_{i j}\right]_{1 \leq i, j \leq 3}$. The analytical form of cross products $X, Y$ and $Z$ in base $\{x, y, z\}$ is given by:

$$
\begin{aligned}
& X=-x \times \hat{x}=-\operatorname{sk}\left(e_{1}\right) \tilde{R} e_{1}=-\left[0,-r_{31}, r_{21}\right]^{T} \\
& Y=-y \times \hat{y}=-\operatorname{sk}\left(e_{2}\right) \tilde{R} e_{2}=-\left[r_{32}, 0,-r_{12}\right]^{T} \\
& Z=-z \times \hat{z}=-\operatorname{sk}\left(e_{3}\right) \tilde{R} e_{3}=-\left[r_{13}, r_{31}, 0\right]^{T}
\end{aligned}
$$

When adding those three terms, the components of the rotation vector $\omega$ appear (Murray et al., 1994):

$$
X+Y+Z=-\left[\begin{array}{l}
r_{32}-r_{23} \\
r_{13}-r_{31} \\
r_{21}-r_{12}
\end{array}\right]=-2(\sin \alpha) \omega
$$

The condition $X+Y+Z=0$ defining the invariant set becomes:

$$
2(\sin \alpha) \omega=0
$$

$\omega$ being a unit vector, this is only possible if $\alpha=0$ or $\alpha= \pm \pi$. This means that $\hat{R}$ can converge to the real attitude matrix (case $\alpha=$ 0 ) or to a matrix deduced from the real one by an axial symmetry about axis $\omega$ (case $\alpha=$ $\pm \pi$ ). To guarantee the convergence of $\hat{R}$ to $R$, we need to characterize the attractive domain of the equilibrium point $\alpha=0$. Initializing the observer state inside this domain will ensure the convergence to the real attitude matrix. First, let us recall the relation between the rotation angle $\alpha$ and $\tilde{R}$ (Murray et al., 1994):

$$
\cos \alpha=\frac{\operatorname{trace}(\tilde{R})-1}{2}
$$

The expression of the Lyapunov function given by (10) can be written as follows:

$$
V=2(1-\cos \alpha)+\frac{1}{2} \Gamma^{-1}|\tilde{b}|^{2}
$$

${ }^{5} \forall u, v \in \mathbf{R}^{3}, \operatorname{sk}(u)^{T}=-\operatorname{sk}(u)$ and $\operatorname{sk}(u) v=-\operatorname{sk}(v) u$
Let us define $\mathcal{D}_{a}=\left\{(\alpha, \tilde{b}) \in \mathbb{R} \times \mathbb{R}^{3} / V(\alpha, \tilde{b}) \leq\right.$ $a\}$ the region delimited by the equipotential curve $\{V=a\}$. It is clear that for $\forall v, w \in \mathbb{R} / 0 \leq v \leq$ $w, \mathcal{D}_{v} \subset \mathcal{D}_{w}$ and that $\forall a \geq 0,(\alpha, \tilde{b})=(0,0) \in$ $\mathcal{D}_{a}$. As $\dot{V}<0$, we ensure that $\mathcal{D}_{V(t)} \subset \mathcal{D}_{V(0)}$. To determine the attractive domain of the point $(\alpha, \tilde{b})=(0,0)$, it is sufficient to consider the biggest region $\mathcal{D}_{a}$ within the hyperplanes $\alpha= \pm \pi$.

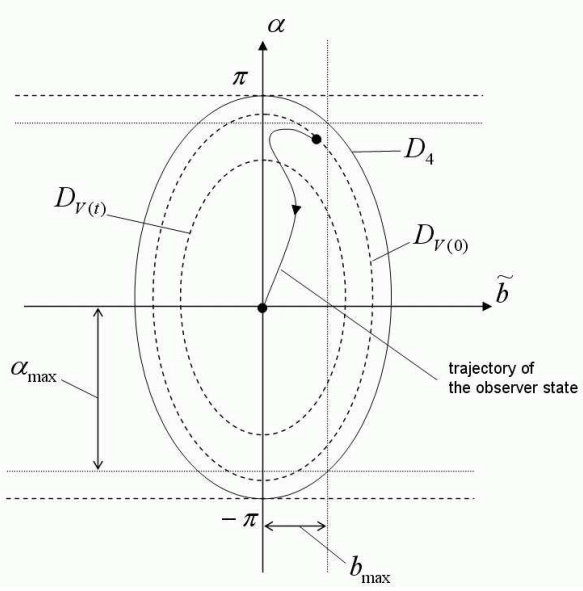

Fig. 3. Illustration of the attractive domain around equilibrium configuration $\alpha=0$.

This region is tangent to both hyperplanes at points $(\alpha, \tilde{b})=( \pm \pi, 0)$. At these points, $V=$ $2(1-\cos ( \pm \pi))=4$. The attractive domain is the interior of $\mathcal{D}_{4}$. Considering the the upper born on the initial gyroscope's bias estimation error $\tilde{b}_{\max }$, the maximal initial angular gap $\alpha_{0}$ allowed can be determined by the relation:

$$
V(0)=2\left(1-\cos \alpha_{0}\right)+\frac{1}{2} \Gamma^{-1}\left|\tilde{b}_{0}\right|^{2} \leq 4
$$

This leads to the upper born on $\alpha_{0}{ }^{6}$ and $\tilde{b}_{0}$ given by (9). The observer being initialized in this way, the convergence of $\hat{R}$ to $R$ is ensured. $\hat{R} \rightarrow R$ means $\tilde{R} \rightarrow I_{3 \times 3}$ and $\dot{\tilde{R}} \rightarrow 0$. Furthermore, the convergence to zero of $X+Y+Z$ means that $\varpi$ will tend to zero, as well as $\dot{\hat{b}}$ (cf. (7) and (8)). Therefore, the estimation error on gyroscope's bias tends to a constant value denoted by $\tilde{b}^{\infty}$. When $t \rightarrow \infty$ in the expression of $\dot{\tilde{R}}$ given by (11), it can be shown that $\tilde{b}^{\infty}$ verifies:

$$
\operatorname{sk}\left(\tilde{b}^{\infty}\right)=0
$$

which implies the convergence of $\tilde{b}$ to zero. $\square$

\section{SIMULATION RESULTS}

In our simulation, the observer given by the equation (5) is sampled using Rodrigue's formula:

$$
\hat{R}_{k+1}=\hat{R}_{k} A_{k}
$$

\footnotetext{
6 Taking an adaption gain very small $\Gamma=0.01$, and an important initial error on gyroscope's bias estimation $b_{\max }=5^{\circ} / \mathrm{s}$, one gets $\alpha_{\max } \approx 145^{\circ}$. In practice, initializing the observer state with the first measured attitude matrix, maximal angular gap $\alpha_{0}$ will not exceed $30^{\circ}$ so this upper born is not very constraining
} 
where $A_{k}=\exp \left(\operatorname{sk}\left(\omega_{k}\right) \tau\right)$, given that $\Omega(t)=\omega_{k}$ for $t \in[k \tau,(k+1) \tau]$. $A_{k}$ has a closed form solution (Murray et al., 1994) of the form:

$A_{k}=I_{3 \times 3}+\frac{\sin \left(\left\|\omega_{k}\right\| \tau\right)}{\left\|\omega_{k}\right\|} \operatorname{sk}\left(\omega_{k}\right)+\frac{1-\cos \left(\left\|\omega_{k}\right\| \tau\right)}{\left\|\omega_{k}\right\|^{2}} \operatorname{sk}^{2}\left(\omega_{k}\right)$

This discrete form will preserve the evolution of $\hat{R}_{k}$ within $S O(3)$ because $A_{k}=\exp \left(\operatorname{sk}\left(\omega_{k}\right) \tau\right)$ is a rotation matrix. However, to prevent any accumulation of numerical errors, columns of $\hat{R}_{k}$ can be kept orthogonal by Gram-Schmidt algorithm.
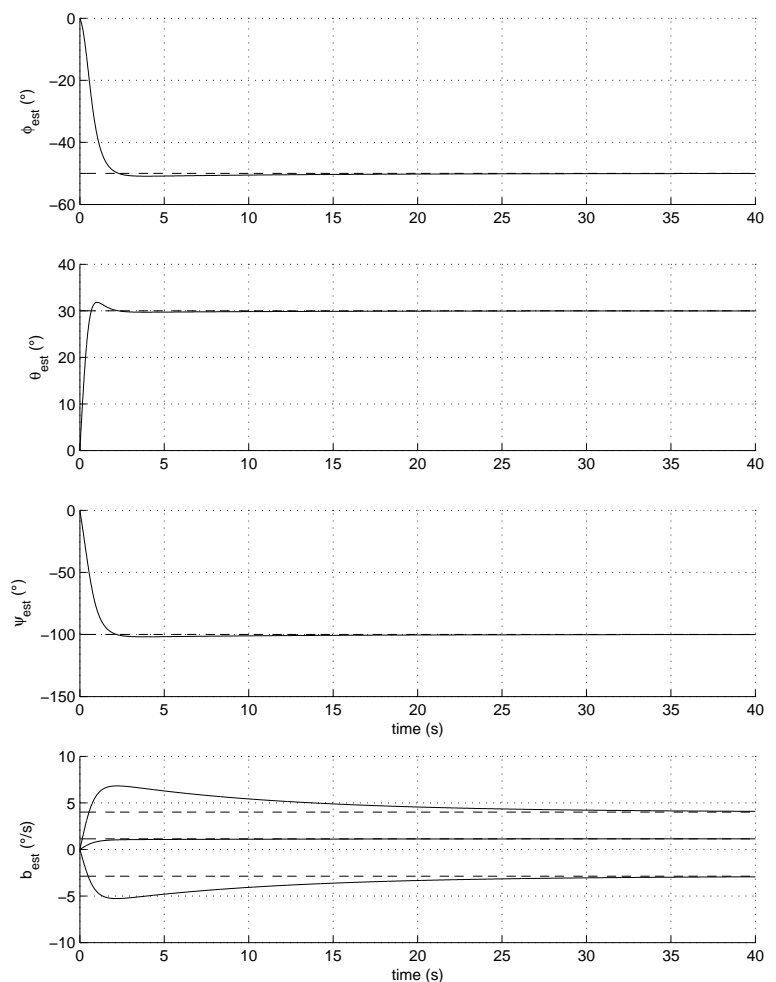

Fig. 4. Evolution of the estimated Euler angles and gyroscope's bias.

Simulations were performed to represent the convergence of the three Euler angles estimation and the gyroscope's bias estimation. A good behavior of the estimator can be observed on figure 4 in spite of large gaps between initial values of estimated Euler angles and the real ones $\left(\left[-50^{\circ}, 30^{\circ},-100^{\circ}\right]\right)$ and an important gyroscopic drift $\left(b=[0.02,-0.05,0.07]^{T} \mathrm{rd} / \mathrm{s}\right)$.

\section{CONCLUSION}

In this draft, we have proposed an observation strategy to estimate the orientation of a VTOL UAV taking advantage from both short term precision given by integration of measured angular rates and long term precision provided by accelerometers and magnetometers. A nonlinear estimation law defined on $S O(3)$ and an adaptive filter on gyroscope's bias have been designed and a theorem both proves the convergence of the method and provides an upper born on the initial angular gap between the estimated orientation matrix and the real one. Behavior obtained in simulation is very encouraging and integration inside the embedded calculator of Bertin Technologies VTOL UAV is in progress. At this moment, a similar but simplified algorithm providing roll and pitch angles has been successfully integrated and allows remote controlled flight using PIDs. In further work, we will propose a nonlinear control law coupled with this estimation law which ensures the stability of the closed loop system despite wind gusts.

\section{ACKNOWLEDGMENTS}

The authors wish to express their gratitude to the French Ministry of Research and Technology and the National Direction of Armament DGA for granting this research program. They also thank Olivier Philippe, VTOL UAV project manager at Bertin Technologies, who provided funding and support in this research. They also address a special thanks to the whole drone team of Bertin Technologies for their assistance and advices.

\section{REFERENCES}

Boiffier, J.L. (1998). The Dynamics Of Flight. Jhon Wiley \& Sons.

Grewal, M.S., L. Weill and A.P Andrews (2001). Global Positioning Systems, Inertial Navigation, and Integration. Jhon Wiley \& Sons.

Hamel, T., R. Mahony, R. Lozano and J. Ostrowski (2002). Dynamic modelling and configuration stabilization for an x4-flyer. In: Proc. of the 15th International Federation of Automatic Control Symposium, IFAC 2002. Barcelona, Spain.

Krstic, M., I. Kanellakopoulos and P. Kokotovic (1995). Nonlinear and Adaptive Control Design. John Wiley \& Sons.

Marins, J.L, X. Yun, E.R. Bachmann, R.B. McGhee and M.J. Zyda (2001). An extended kalman filter for quaternion-based orientation estimation using marg sensors. In: Proc. of the IEEE Int. Conf. on Intelligent Robots and Systems. Maui, Hawaii, USA. pp. 2003-2011.

Murray, R.M., Z. Li and S. Sastry (1994). A Mathematical Introduction to Robotic Manipulation. CRC Press.

Pflimlin, J.M., P. Souères and T. Hamel (2004). Hovering flight stabilization in wind gusts for a ducted fan uav. In: Proc. of the 43rd IEEE Conference on Decision and Control, CDC04. Atlantis, Bahamas.

Radix, J.C. (1980). Systèmes inertiels à composants liés "strap-down". CEPADUES Editions.

Suh, Y. Soo (2003). attitude estimation using low cost accelerometer and gyroscope. In: Proc. of the 7th Korea Russia International Symposium, KORUS03. Ulsan, Korea. pp. 423-427. 